\title{
Mapping allosteric transitions of an enzyme with SAXS, cryo-EM, and crystallography
}

\author{
William C. Thomas ${ }^{1,2}$, F. Philip Brooks III $^{2}$, Audrey A. Burnim ${ }^{1}$, John-Paul
} Bacik $^{1}$, JoAnne Stubbe ${ }^{3}$, Jason T. Kaelber ${ }^{4}$, James Chen ${ }^{5}$, and Nozomi Ando ${ }^{1}$

1. Department of Chemistry \& Chemical Biology, Cornell University

2. Department of Chemistry, Princeton University

3. Department of Chemistry, Massachusetts Institute of Technology

4. Institute for Quantitative Biomedicine, Rutgers University

5. Department of Biochemistry \& Molecular Biology, Oregon Health and Sciences University

\begin{abstract}
:
Allosteric enzymes present a unique challenge to structural characterization because many of their underlying mechanisms are rooted in dynamic conformational change at the tertiary and quaternary levels. One such class of conformationally mutable enzymes is the ribonucleotide reductase (RNR) family, which uses a conserved, radical-based mechanism to catalyze the de novo conversion of ribonucleotides to deoxyribonucleotides. Due to their central role in DNA metabolism, RNRs have evolved complex and varied modes of regulation that frequently involve dramatic transitions between active and inactive oligomeric states upon binding of nucleotide effectors. A combination of SAXS nucleotide titration experiments and chromatography-coupled SAXS were used to map out the conformational landscape of a newly discovered variant of allosteric regulation found in a subset of bacterial RNRs, revealing the reversible interconversion of six unique oligomeric structures. These SAXS studies in turn enabled a hypothesis-driven approach to structure determination by crystallography and cryo-electron microscopy that yielded structures of a catalytically important intermediate and two novel, inhibited helical filaments. Combined, these studies highlight the conformational gymnastics necessary for RNR activity and the molecular basis for its control via an evolutionarily convergent form of allostery.
\end{abstract}

Contact Information:

William Thomas wct44@cornell.edu

Phil Brooks fbrooks@alumni.princeton.edu

Audrey Burnim aab293@cornell.edu

John Bacik jpb392@cornell.edu

JoAnne Stubbe stubbe@mit.edu

Jason Kaelber jason.kaelber@rutgers.edu

James Chen chezh@ohsu.edu

Nozomi Ando nozomi.ando@cornell.edu 